\title{
VI,
}

Aus Prof. Flechsig's Laboratorium der psychiatrischen und Nervenklinik der Universität Leipzig.

\section{Beitrag zur Kenntniss der hereditären Ataxie und Tleinhirnatrophie.}

\author{
Von \\ Dr. med. P. Menzel \\ in Hainitz-Grosspostwitz bei Bautzen. \\ (Hierzu Taf. V.)
}

\begin{abstract}
N
achdem im Jahre 1863 Friedreich die ersten Fälle der nach ihm benannten Form der Tabes, der hereditären Ataxie, beschrieben hatte, sind in der Folge eine grosse Anzahl gleicher Erkrankungen bekannt geworden, and im Anschluss daran wurden verschiedene, zum Theil von einander abweichende Ansichten über das Wesen dieser Krankheit laut, die sich theilweise auf Sectionsbefunde stützten.

Während einige Autoren den Process als eine diffuse oder multiple Sclerose der Medulla spinalis und Medulla oblongata ansahen (Féré, Seguin ef. No. 37 und Nr. 63 des Literaturverzeichnisses), Hammond (cf. No. 41) geneigt war, die Medulla oblongata als deu primären Sitz anzusehen mit anschliessender Betheiligung des Cerebellum, und Charcot (cf. Nr. 78) noch neuerdings die hereditäre Ataxie in die Mitte zwischen Tabes dorsalis und multiple Sclerose stellt, wurde zuerst von Kahler und Pick (cf. No. 17 und No. 28) der Charakter der Krankheit als der einer combinirten Systemerkrankung auf Grund einer hereditär vorhandenen Schwäche und Entwicklungshemmung der spinalen Fasersysteme bezeichnet; sie nehmen ein Stehenbleiben auf einer gewissen Stufe der Markscheidenbildung der später intensiver erkrankenden Fasersysteme an. Im
\end{abstract}


Wesentlichen diesen sieh anschliessend, nehmen Friedreich (cf. No.12) und Schultze (cf. No. 39) eine Entwicklungshemmung im Rückenmarke und Praedisposition zu sclerotischen Erkrankungen an; weder die Atrophie des Bulbus medullae noch die Rückenmarksdegeneration sei secundär, sagt Friedreich, vielmehr scheine eine völlige Ausbildung und Entwicklung der Medulla spinalis und oblongata nicht stattgehabt zu haben. Und dieser Annahme einer combinirten Systemerkrankung auf hereditärer Grundlage sind zahlreiche andere Autoren beigetreten: Leyden (cf. No. 47), Seeligmüller (cf. No. 82), Smith (cf. No. 74), Rütimeyer (cf. No. 86) u. a.

Von einer Anzahl von Fällen sind die Sectionsbefunde veröffentlicht worden, und diese stimmen darin überein, dass es sich hier um degenerative Processe in den Hintersträngen, Pyramidenseitenstrangbahnen, Kleinhirnseitenstrangbahnen und Clarke'schen Säulen bandelt; so bei Kahler und Pick (cf. No. 17, 28), Friedreich (cf. No. 2, 11, 12), Scbultze (cf. No.25, 39, 51), Charcot (cf. No.68, 78, 92), Longuet (No. 57, 69), Ferrier (No. 89) Rütimeyer (No. 48, 86, 98), Smith (No. 74), Erlicki und Rybalkin (No.65, 80), Borgherini (No. 99) u. a. In einigen Fällen wurde die Erkrankung bis in die Medulla oblongata verfolgt: Friedreich, Rütimeyer, Brousse (cf. No. 36).

Ist schon durch diese Untersuchungen das Wesen der hereditären Ataxie häufig genug behandelt, so dürfte es doch vielleicht nicht überflüssig sein, über einen Fall zu berichten, der in mehrfacher Beziehung einige Besonderheiten darbietet.

Derselbe kam in der Districtspoliklinik des Herrn Prof. Heabner in Leipzig zur Beobachtung, und das Sectionsmaterial wird im Laboratorium der Irrenklinik daselbst aufbewahrt. Ich benutze mit Freude diese Gelegenheit, Herrn Prof. Heubner für gütige Ueberlassung der Krankengeschichte und Herrn Prof. Flechsig für vielfache Anregung und Unterstützung bei der mikroskopischen Untersuchung meinen Dank auszusprechen. Ein Theil der Schnitte durch das Gehirn ist von Dr. Bogroff in Odessa angefertigt worden.

\section{Krankengeschichte.}

Robert Schweigel, geb. 1840, Goldarbeiter, beim Tode 46 Jahre alt.

Der Vater des Patienten war gesund, starb 70 Jahre alt an Gehirnschlag.

Die Mutter desselben soll in der Jugend ganz gesand gewesen sein, dick und stark und soll von Gesundheit gestrotzt haben. Später aber \#habe sie etwas bekommen", habe forlwährend mit dem Kopfo geschüttelt, einen ganz 
taumeligen und schliesslich ganz unsicheren Gang gehabt, so dass sie Jahre lang vor ibrem Tode nicht mehr auf die Strasse gekommen sei. Sie starb im Alter von 60 Jahren.

Ueber die Geschwister des Patienten wird Folgendes in Erfahrung gebracht (durch die Eran desselben):

Der älteste Bruder des Patienten ist jetzt hoch in den 40 ger Jabren, lebt in Hamburg und soll ganz gesund sein.

Ein zweiter Bruder wurde während der Militärzeit bereits krank und bat dann über zwanzig Jahre gesiecht. Er litt an „Rückenmarksverzehrung “, bat auch "geschüitelt", starb einige 40 Jahre alt.

Ein dritter Bruder lebt in Naumburg; diesor ist schwach im Kopfe, bat aber keinen taumelnden Gang, keine schüttelnden Bewegungen.

Eine äliere Schwester wurdo ungefähr in Milte der 30 ger Lebensjahre krank. Sie bekam einen "tapplichen" Gang und war nicht im Stande, den Kopf still zu halten, "der ibr fortwährend auf die Seite flog;" wenn sie ron Halle, wo sie wohnte, zu Besuch kam, sah sie aus, wie eine Wahnsinnige; sie nahm sich im Jahre 1878 das Leben.

Eine zweite Sobwester lat sich im Sommer 1885 das Leben genommen im Alter von 36 Jahren. Sie war eine starke, gesund aussehende Person mit weisser Haut, rothen Wangen; aber sio batte etwas Unsicheres in ihrem Tritt, fiel leicht hin und wurde auch allmälig schwächer; als man sie wegen nicht mehr vorhandener Arbeitsfähigkeit in eine Anstalt bringen wollte, lief sie in's Wasser.

Eine dritte Schwester, die jüngste, ist in Berlin verheirathet; dieselbe ist zur Zeit "resolut", aber auch sie ist nervenschwach.

Also von 7 Geschwister sind 6 nicht normal in Bezug auf ihr Nervensystem. Die zwei Schwestern und vielleicht der eine Bruder waren in analoger Woise afficirt wie Patient selbst.

Der Kranke verlegte den Beginn seiner Krankbeit in das Jahr 1874, wo das Loiden mit Unsicherheit und Schwäche beim Gehen und mit Kreuzschmerzen begonnen haben soll. Die Frau bemerkte aber schon vor der im Jahre 1868 erfolgten Verheirathung Abnormitäten an demselben.

Als Knabe soll er gesund gewesen sein, habe sich normal entwickelt, und er habe - berichtet die Frau nach den Erzählungen des Mannes - bis zum 17. Jahre eine sebr schöne Handschrift gesehrieben. Schon von da an aber sei ihm das Scbreiben schwer geworden; er schrieb nicht mehr gern. In der Zeit der Verbeirathung $(1868,28$ Jahre alt) schrieb er schon ganz "krakelig". Seit dem Jahre 1876 war das Schreiben überhaupt gar nicht mehr möglich.

Er war ein geschickter Goldarbeiter. Im Anfange nach der Verheirathung machte er auch feine und kleine Arbeiten noch durchaus gesehickt, aber die Fran merkte, dass es ihm schwer wurde, als ob er ungewöhnliche Hindernisse überwinden müsse. Ganz allmälig verscblechterte sich diese Fühigkeit zu arbeiten. Seit 1880 konnte er absolut nichts mebr arbeiten, er konnte nicht einmal die Reparaturen mehr richtig beschreiben. 
Als junger Mann soll er ein unverwüstlicher Tänzer gewesen sein; schon während der Verlobungszeit (1868) aber fing er an, weniger geru zu tanzen; er sagte: es würde ihm schwindlig beim Tanzen. Schon damals bemerkte die Frau, dass er es gern hatte, wenn man ihn führte, dass er manchmal mit dem rechten Beine stark nach auswärts schleuderte, und dass er, wenn sie sich führten, ihr öfters zwisohen die Beine "quergelte". - Schon als junger Ehemann blieb er gern zu Hause, Abends ging er ohne Frau nicht aus, weil schon damals der Gang im Dunkeln ganz hochgradig schlecht, schwankend und tanmelnd war. Von Mitte dor 70 ger Jahre an ging er immer schlechter und wollte gar nicht mehr auf die Strasse, woil die Leute ihn auf seine sonderbaren Bewegungen hin ansahen. Er fuhr dann nur noch Sonntags aus. Seit $1884 \mathrm{kam}$ er überhaupt nicht mehr auf die Strasse.

Sehon seitdem die Frau den Mann kennt, ist er im Dunkeln äusserst ungeschickt. Er konnte schon im Beginn der Ehe im Dunkeln keine Lampe anzünden; wollte er bei Gelegenheit der ersten Entbindung seiner Frau (1869) Nachts etwas helfen, so stürzte er sehr oft aus dem Bette und fiel in der Stube hin, fand nicht, wonach or suchte $u$. dgl.

Aber auch am Tage griff er schon in den ersten Ehejahren öfters daneben, wenn er etwas in die Hand nehmen wollte. Das Essen warde dem Manne schwer, so lange die Frau sich erinnern kann: er stach sich öfter mit der Gabel in den Mund; fuhr mit dem Bissen neben dem Munde vorbei, so dass er manchmal ganz wüthend wurde und die Gabol weit von sich weg warf. Eir ass schon seit Jahren desbalb nicht mit der übrigon Familie, sondern allein für sich. In den letzten Lebensjabren musste ihn seine Frau ganz füttern. Fisch bat die Frau nie bringen dürfen, weil er die Gräten nioht ordentlich zu eliminiren im Stande war. Im letzten Lebensjahre konnte er überhaupt Festes nicht mehr gat schlucken.

Die Stubl- und Urinentleerung war bis zuletzt pollkommen ungestört.

Ebenso konnte der Coitus ausgeführt werden, Frau S. wurde noch im Jahre 1884 gravida, es trat aber Fehlgeburt ein.

Subjective sensible Störangøn bot er nie viel dar. In den Jabren 1874 bis 1881 litt or an anfailswoise auftretenden heftigen Kreuzschmerzen und von da ab an Schmerzen im Nacken. Letztere bestanden nicht eigentlich in heftigem Stechen als in einem Gefühl des Geschwollenseins.

Psychische Functionen waren nicht anffällig gestört. Allerdings war Patient ron Aufang der Ehe an immer sehr still für sich und ruhig. Die Frau weinte anfangs häufig daräber, dass er so sehr in sich gekehrt war and so wenig sprach.

Das Gedächtniss war und blieb stets gut, er konnte gut rechnen und dergl.; über Kopfschmerzen hat er niemals geklagt.

Seit ungefähr 1879 ist die Sprache unbeholfen und schwerfällig geworden und fühlt Patient eine wirkliche Schwäche im rechten Arme neben der schon längst bestehenden Unsicherbeit der Bewegungen.

Er wurde von einer grossen Zabl von Aerzten behandelt; meist wurde die Diagnose auf multiple Sclerose gestellt. 
Im Juli 1882 sab ich den Kranken zum ersten Male und nabm dabei den folgenden Status auf:

Nicht gerade unlräftiger, mehr magerer, aber zienlich muskulöser Körper ron graubleicher Färbung. Es fällt sofort auf, dass Patient den Kopf nach rechts gedreht bält; und dass sich diese Drehung bei jeder intendirten Bewegung fortwährend vermehrt. Steht er auf und macht einige Schritte, so wirft er beinahe bei jedem Schritte den Kopf etwas weiter herum, so dass er ganz nach rechts und schliesslich fast nach rechts rückwärts sieht; er hat dabei die Empfindung, als ob der Nacken anschwölle. Die Drehung beruht auf einer krampfhaften, immer stärker werdenden Contraction des linken M. sternocleidomastoideus. Um ihr einigermassen entgegenzuwirken, legt er die Hand an die linke Nacken- und Halsseite. Dasselbe tritt ein, wenn or im Sitzen zu lesen versucht; unwillkürlich wenden sich der Kopf und die Augen immmer weiter nach rechts, so dass er das Buch immer mebr nach rechts bringen muss, es schliesslich ganz rechtsseitig hält und die Augen ganz in den rechten Winkeln stehen. Hält man den Kopf fest, so kann er auch mit gerade gerichtetem Blicke ganz gut lesen; überhaupt ist die Gesichtsschärfe sowie auch die Bewegung der Angen nach allen Richtungen hin ganz normal.

Auch im Gesicht zeigen sich krampfhafte Mitbewegungen: Erstlich reisst Patient während des Gehens und Lesens in fortwährender Progression die Augen immer weiter auf, so dass das Gesicht schliesslich den Ausdruck des grössten Schreckens oder Staunens annimmt.

Zweitens zeigt er eine Vertiofung der Nasolabialfalte und eine Contraction der Muskeln des Mundes und Kinnes, wie man sie nur beim schwersten Tetanus sonst sieht, und auch dieser ganz ungewöhnliche mimische Ausdruck des unteren Gesichtes wird immer ausgeprägter, je länger Patient intendirte Bewegungen rornimmt.

Drittens endlich zeigt sich Analoges an der Sprache. Dieselbe ist eigenthümlich mühsam, gepresst, abgesetzt, als ob es ihm Mühe machte, das Wort herauszubringen, ungefähr wie ein von höchst intensiven Leibschmerzen gepeinigter Mensch spricht; dabei aber ist das einzelne Wort durohaus scharf articulirt. Dieses Hervorstossen der einzelnen Worte wird immer stärker und dabei in sehr auffallender Weise die Sprache immer lauter, je länger er spricht, also jo zahlreiohere Impulse or verwendet hat.

Alle diese eigenthümlichen Krampfbewegungen werden sofort viel milder und geringer, wenn man den Patienten eine Lage einnehmen lässt, welche möglichst wenig motorische Impulse in Anspruch nimmt. Lässt man ihn, den Oberkörper und Kopf völlig unterstützt, eine möglichst zwanglose Stellung im Bette einnehmen, so verschwindet sogleich die tetanische Nasolabialfalte, die Augen werden nicht mehr so aufgerissen, er kann bei gerader Kopfhaltung lesen; auch dieSprache wird weniger gepresst, und er kann sogar eineu Buchstaben mit Mühe schreiben.

In aufrechter Stellung ist letzteres absolut unmöglich; denn es besteht die hochgradigste Ataxie beider oberen Extremitäten. Jede Bewegung geschieht im Zickzack, schleudernd, an den gesuchten Gegenständen vorbei etc. 
Knöpfen, Binden, selbst Essen ist ganz unmöglich. Dieselbe Ataxie besteht in den unteren Extremitäten: stampfender, schleudernder, unsicberer Gang. Bei geschlossenen Augen sofortiges Umfallen; sohon boi blossom Umdrehen mit offenen Augen Sehwindel. Bei jedem Gang durch's Zimmer muss Patient sich an den Möbeln anhalten. Die rechte Körperbälfte scheint dabei noch etwas stärker atactisch als die linke.

Die rohe Kraft der oberen Extremitäten ist deutlich abgeschwächt, dabei aber rechts wieder mobr als links.

Auch beim Versuche, auf einem Beine zu stehen, zittert das rechte viel stärker als das linke. Die rohe Kraft des Quadriceps femoris erscheint beiderseits unversehrt and gleioh, ebenso diejenige der Beugemuskeln am Oberschenkel.

Die Pupillen sind eber weit als eng; sie reagiren sowohl bei Lichteinfall wio bei Accommodation schwach.

Die Patellarsehnenreflexe sind eher etwas gesteigert.

Die Sensibilität an den Armen und Beinen ist für die Berübrungen vollkommen unversebrt. Die feinsten Berührungen werden dentlich empfunden; Streicheln, Kneipen, Stechen auf's schärfste überall unterschieden; jede Figur, die ihm auf Hand oder Fuss gemalt wird, wird sogleich richtig erkannt.

Der hier geschilderte Status erlitt von 1882 an, unter meiner - allordings oft monatelarg unterbrochenen - Beobachtung eine ganz langsame und allmälig sich rollziehende Verschlimmerung, wobei Zeiten vorkamen, in denen Patient, freilich subjectiv, auch sich besser fühlte. - So war er z. B. im September 1882 recht zufrieden, gab an, seine Glieder seien geschmeidiger geworden, war $a b$ und zu doch mal wieder ausgegangen u. dgl.

In den Jahren 1883 und 1884 wurde selten Hülfe in der Distriots-Poliklinik gesucht. Der Zustand bot keine plötzlichen Veränderungen ader Ereignisse, und ich habe in diesen beiden Jahren keine Notizen über den Patienten gemacht.

Im Herbste 1885 steigerten sich die Besehwerden des Patienten wieder mehr, und namentlich klagte er über grosse Sohlaflosigkeit, eine Erscheinung, die neu hinzugetreten war.

Mitte November 1885 wurde wieder ein Status aufgenommen: Patient sieht viel soblechter aus als vor drei Jahren, ist bedeutend abgemagert, die Hautfarbe geradeza cachectisch.

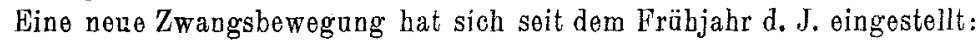
sehr häufig reisst jetzt Pationt den Mund ganz weit auf, wio beim Maximum des Gähnens, und an diese Bewegung schliessen sich dann mehrfache wiederholte Kaubewegungen an. Dies geschieht, ehe Patient zu sprechen anfängt, wenn er sich aufrichtet etc. Patient ist höchstens im Liegen dann und wann im Stande, diese krampfhafte Bewegung zu unterdräcken.

Auch im rechten Arme bekommt Patient öfter anwillkürliche Stösse, die zu einer choreaartigen Bewegung führen.

Die Sprache ist noch viel sohwerer. Nach langem Aufreissen des Mundes, vielfachen Kaubewegungen, mehrmaligem Ansetzen bringt or endlich 
stossweise ein Wort hervor; auch dies ist aber nicht mohr so dentlich wie früher, sondern weniger gut articulirt; die Stimme ist dabei sehr laut, last überanstrengt.

Der rechte Arm zeigt deutlich grössere Schwäche als der linke,

Beim Gehen kommt er geradeaus gar nicht mehr vorwärts, woil's ihm jedes Mal sofort den Kopf nach der rechten Seite herumwirft. In der Stube hilft er sich aber noch, sich anhaltend, herum.

Im Hinterkopf hat er ein beklommenes Gefühl, in der rechten Schulter die Empfindung des Angeschwollenseins.

Februar 1886. Während des Winters hat sich der Zustand immer mehr verschlechtert. Seit etwa Mitte Februar liegt Pationt fest, "weil die Beine nicht mehr fortwollen". Beim Versuch zu gehen, geräth or sofort in Gefahr umzufallen. Sehr viel Schwindel. Des Nachts fällt or oft aus dem Bett. Dabei sind die Beine hochgradig abgemagert, alle Muskeln derselben aber in abnormer Spannung, deutlich unter der fettlosen Haut sich abhebend.

Auch in den Armen vielfach abnorme Spannungen; hat or etwas angefasst, so kann er es nicht wieder loslassen.

Die Patellarreflexe deutlich, aber nicht mehr erhöbt.

Am 1. März wurde nochmals die Sensibilität an den unteren Extremitäten geprüft. Borührung, ebenso Sohmerz wird überall ganz deutlich empfunden; jede Empfindung wird richtig localisirt.

Gesicht and Gehör gut; Bewegung der Bulbi frei.

Im Laufe des März immer mangelhaftere Nahrungsaufnahme, zunehmende Abmagerung.

Am 6. April 1886 kann Patient kaum mehr reden, ist offenbar etwas benommen. Beine in Beugecontractur, Hände in Krallenstellung; die 2. und 3. Finger beiderseits in Hyperextension; Gelenke zwischen 1. and 2. Phalanx nach vorn durchgedrückt. Dor Mund steht weit offen, eingeschüttete Milch wird noch gut geschluokt.

7. April 1886 früh 4 Uhr erfolgte der Tod.

Section eodem die, Nachmittags 2 Uhr.

Enorm abgemagerte Leiche. Beine in Beugecontractur. Geringer Decubitus. Schädel sehr dick, asymmetrisch. Dura mater schlaff, ohne Hämatom. - An den herausgenommenen nervösen Centralorganen wird sofort die ganz hohgradige Atrophie des Kleinhirns und der Brücke wahrgenommen und daher Gehirn und Räckenmark zunächst ohne eingehendere Untersuchung aufbewahrt.

Von der übrigen Section ist noch folgendes hervorzuheben:

In der rechten Lungenspitze befindet sich eine sohiefrige Induration mit narbiger Einziehung und geringer Verwachsung. Der untere Theil des rechten unteren Lappens ist brandig zerfallen, stark stinkend; die Umgebung der Brandherde infiltrirt, yon kleinen eitrigen Herden durchsetat. Auch im linken Oberlappen eine Pigmentinfiltration; der ganze untere Lappen grösser, derb, luftleer, von kloinen eitrigen und brandigen Herden durchsetzt. 
Herzfleisch blass, schlaff, Klappen und grössere Gefässe normal. Leber normal gross, Galle dunkel, dickflüssig. Hilz schlaff, blass. Nieren vor normaler Consistenz, Grösse und Farbe. Magen und Darm ohne auffällige Abnormitäten. In der Blase klarer Urin.

Kinder des R. Schweigel:

1. 1869 geboren, starb jung,

2. 1871 geborenes Mädchen, gesund,

3. 4. jung gestorben,

5. 1876 geborenes Mädchen, gesund,

6. 1878 geborene Lonise S. mit hereditärer Ataxie behaftet,

7. 1879 geborenes Mädchen, gesund.

Vergleicht man dieses Krankenbild mit dem von Friedrejeh angegebenen Symptomencomplex - Romberg'sches Symptom, Ataxie, meist atactischer Nystagmus und Sprachstörung, Fehlen des Patellarreflexes, Fehlen der lancinirenden Schmerzen und Parästhesien, Erhaltung der Hautsensibilität und des Muskelsinnes, Intactheit der Sphinkterenfunction, fehlende Neigung zur Decubitusbildung und Fehlen des Robertson'schen Symptomes -, so ergiebt sich eine grosse Uebereinstimmung beider, sodass die Diagnose der Friedreich'schen Tabes in unserem Falle wohlbegründet erscheint.

Die lange Daner der Krankheit, der frühzeitige Beginn - erste Zeichen im 17. Lebensjahre - entsprechen den Beobachtungen des ersten Autors; bei der Tochter des S. übrigens traten die ersten Erscheinungen schon in den Kinderjahren auf, wie dies auch von $\mathrm{Rüti-}$ meyer (cf. No. 48 und No. 86 des Literaturverzeichnisses) und Wälle (cf. No. 59) beobachtet ist.

Zuerst zeigte sich bei S. Unsicherheit beim Schreiben, und bald stellten sich danach Ataxie aller Extremitäten und das Romberg'sche Symptom ein sowie die auffallende Sprachstörung, Erscheinungen, die sich bis zum Tode des Patienten mehr und mehr steigerten, und zu denen sich allmälig verschiedene $Z_{\text {wangsbewegungen gesellten. }}$

Nystagmus wurde nie beobachtet; derselbe feblte auch in einigen Fällen von Friedreich, von Gowers (cf.31) und Erlicki (cf. No.65).

Das bei den meisten Erkrankungen dieser Art constatirte Fehlen der Patellareflexe blieb jedoch hier aus, im Anfange waren dieselben sogar eher etwas gesteigert, ein Verhalten, welches auch von Seeligmüller und Botkin (cf. No. 62) angegeben wurde.

Die Hautsensibilität blieb stets intact; von anderen Erscheinungen der sensiblen Sphäre wurden nur im Anfange Kreuzschmerzen angegeben und später das Gefühl des Geschwollenseins im Nacken und Hinterkopfe. 
Wie auch sonst in weit vorgeschrittenen Fällen von Friedreich'scher Tabes zur Beobachtung kam (Rütimeyer, Wälle), fanden sich schliesslich bei S. Contracturen der Beine und Hände.

Behufs einer genauen mikroskopischen Untersuchung wurden Rückenmark und Gehirn in Müller'scher Lösung und Alkohol gehärtet und alsdann Schnittreihen aus verschiedenen Höhen des Rückenmarkes bis binauf in die Gegend des Linsenkernes - theils von Dr. Bog ruff in Odessa, theils vom Verfasser - angefertigt, welche nach verschiedenen Tinctionsmethoden behandelt wurden. Es wurden zum Färben verwandt: Alauncarmin, Picrocarmin, Carminammoniak, Lithioncarmin, Safranin, Nigrosin, und die besten Präparate wurden durch Ánwendung des Weigert'schen und des verbesserten Pal 'schen Verfahrens erlangt.

Die Untersuchung ergab folgende Befunde:

Lumbarmark: Grösse 8,7:6,5 Mm. Degeneration der Hinterstränge and Pyramidenseitenstrangbahnen. Die geschwundenen Nervenfasern sind durch bindegewebige Balken ersetzt. In den Hintersträngen finden sich erhaltene Nervenfasern in dem zwischen Hinterhorn und hinterer Commissur gelegenen Theile.

Die Peripherie ist frei von Degeneration; ebenso keine Veränderung in dem übrigen Theile der Seitenstränge and in den Vordersträngen.

Die Bindegewebssepta sind rom geradlinigen Verlaufo abweichend zum Theil nach hinten abgebogen.

Die hinteren Wurzeln sind an Nervenfaserzahl sehr verringert.

Die Hinterhörner wurden auf Grund der Lissauer'schen Forsohungen über den Faserverlanf in denselben (cfr. 84 der Literaturangabe) einer eingehenden Untersuchung unterzogen, wonach sich das Folgende herausstellte.

Die Randzone Lissauer's zeigt keinen Schwund von Nervenfasern; einige transversale feine Faserzüge zwischen dem lateralen und dem medialen Theile der Randzone sind deutlich, welche die in die gelatinöse Substanz eintretenden hinteren Wurzelbündel durchsetzen.

Substantia gelatinosa: In der spongiösen Zone derselben sind fast keine markhaltigen Nervenfasern sichtbar; nur wenige direct aus den hinteren Wurzeln kommende Fasern durchsetzen sie und einige Fasern am Rande der Seitenstränge. In der Substantia gelatinosa selbst sind feine Fasern fast ganz geschwunden, erhalten nur wenige grobe Nervenfasern aus den hinteren Wurzeln, keine rom Hinterstrang her eintretend.

Zwischen Substantia gelatinosa und Substantia spongiosa wohlerhaltene Querschnitte markhaltiger grober Faserbündel, die longitudinal verlaufen (aufsteigende Colonnen Clarke's).

In der Substantia spongiosa sind feine Fasern ziemlich zahlreich erhalten, dagegen keine Quersehnitte feiner longitudinaler Fasern zu sehen.

Die Einstrahlungsfasern alı den Hintersträngen in's Vorderhorn, die die 
Spongiosa durebsetzen (Köllicker's innere Wurzelbündel, Frommann's Strahlenfasern) sind vorhanden, ihr Verlauf zum. Theil deutlich bis zu den lateralen Theilon der Vorderbörner zu verfolgen.

Die hintere Commissur weist wenige markhaltige Fasern auf, die vordere enthält zahlreiche feine Fasern.

Der Centralcanal erscheint dicht gefüllt mit körnigen Massen.

Im Vorderhorn sind die grossen Ganglienzellen an Zahl und Grösse vermindert und die vorderen Wurzeln zum Theil atrophisch.

Dio hnteren Wurzelganglien, schon makroskopisch verkleinert, zeigen unter dem Mikroskop an Querschnitten eine beträchtliche Verringerung der Bündel an markhaltigen Fasern. Längsschnitte Jassen an den degenerirten Nervenfasern einen starken Zerfall der Markscheide erkennen, von der nur stellenwoise einzelne dunkel gefärbte Tropfen sichtbar sind, währond die Schwann'sche Scheide unregelmässig streckenweise mit zahlreichen ungefärbten Körnohen erfüllt, an anderen Stellen ihres Verlaufs völlig leer und zusammengesunken ist. Von Axencylindern ist nichts mehr nacbgewiesen. Im umgebenden Bindegewebe finden sich zahlreiche Fettzellen eingelagert.

Unteres Dorsalmark. Grösse 7,7:6,0 Mm. Degeneration der Gollschen und Burdach'schen Stränge, der Pyramidenseitenstrangbahn und der directen Kleinhirnseitenstrangbahn.

In den Hintersträngen sind die Keilstränge am meisten von der Degeneration betroffen, und zwar die Zones radiculaires postérieures Charcot's (Bandelettes externes) bedeutend mebr als das hintere äussere Feld der Keilstränge; in den Goll'schen Strängen sind noch viele Nervenfasern erhalten, so dass sich dieselben - an nach Pal behandelten Präparaten - deutlich vom stark entfärbten Gebiete der Keilstränge abheben.

Wohlerhaltene Nervenfasern finden sich in den Hintersträngen im Winkel zwischen Hinterhorn und hinterer Commissur und am ganzen peripheren Rande.

In den Seitensträngen betrifft die Degeneration, hier schwächer auftretend als in den Hintersträngen, die Pyramidenseitenstrangbahn und directe Keinhirnseitenstrangbahn; seitliche Grenzschicht und übrige Theile der Seitenstränge und das Gebiet der Vorderstränge bleiben frei von der Degeneration.

Die B indegewebssepta auch hier im Bereiche der Hinter- and Seitenstränge zum Theil rom geradlinigen Verlaufe abwoichend.

Die Randzone zeigt dichtgedrängte erhaltene Nervenfaserquerschnitte.

Die hinteren Wurzeln erscheinen etwas verschmächtigt.

Hinterhorn. Die Substantia gelatinosa weist in ihrem der Peripherie der Hinterstränge anliegenden Theile viele feine Nervenfaserquersehnitte erhalten auf, im Uebrigen sehr wenige feine Fasern und einjge grobe Bündel welche sie durchsetzend ron den hinteren Wurzeln zur Substantia spongiosa gehen.

In der spongiösen Substanz sind eine Anzahl orhaltener dicker Querschnitte markhaltiger, grober, längsverlaufender Faserbündel (aufsteigende Colonnẹn Clarke's). In ibrem hinteren Abschnitte sind zahlreiche quer- 
verlaufende Fasern erhalten, die aus den hinteren Wurzeln und Hintersträngen lommen, im vorderen $\Lambda$ bschnitte oin erhaltenes Netzwerk feiner markhaltiger Fasern.

Von den Einstrahlungsfasern in's Vorderhorn, die aus der Wurzelzone der Hinterstränge kommen, sind nur wenige in ihrem Verlaufe zum Vorderhorn zu verfolgen.

Die Grundsubstanz der Clarke'schen Säulen, durch relative Helligkeit vom übrigen Theile der Substantia spongiosa sich abhebond, wird von vielen erhaltenen längsverlaufenden zarten Fasern durchsetzt. Die Zellen der Clarke'schen Säulen zeigen das Bild der fettig-pigmentöser Degeneration, die Kerne derselben sind zum Theil noch sichtbar - besonders an Präparaten, die mit Picrocarmin und nach $\mathrm{Pal}$ gefärbt sind. - An den untersuchten Querschnitten sind weder Einstrahlungsfasern aus den Hintersträngen in die Clarke'schen Säulen erkennbar, noch die Flechsig'schen Kleinhirnbündel; ebensowenig lassen sich Fasern nachweisen, die ans dem Vorderhorn und der Gegend der vorderen Commissur zu den Clarke'schen Säulen verlaufen. Was das zarte Notzwerk feiner markhaltiger Fasern betrifft, das die Zellen der Clarke'schen Säulen umgiebt, so finden sich darin einige Faserzüge aus den Hinterhörnern und Hintersträngen, doch ist nirgends eine Verbindung derselben mit jenen Zellen nachweisbar - ein fraglicher Punkt, auf welchen Lissaner (of. No. 84) in seiner ober citirten Arbeit aufmerksam gemacht hat.

Die hintere Commissur enthält einige erhaltene Faserbündel, die vordere ist reich an feinen und groben Fasern.

Der Centralcanal mit körniger Masse angefüllt.

Im Vorderhorn finden sich zum Theil atropische Ganglienzellen und diesen entsprechend atrophische vordere Wuzelfasern.

Das obere Dorsalmark zeigt dasselbe Verhalten, doch sind die (im untern Theile) intacten Gebiete der Hinterstränge hier kleiner, als im unteren Theile.

Cervicalmark: Halsanschwellung, Grösse: $12,2: 6,5 \mathrm{Mm}$.

In den Hintersrängen findet sich Degeneration der Goll'schen und mehr noch der Keilstränge, wenig afficirt ist der peripbere Rand, intact die Region im Winkel zwisohen Hinterhorn und hinterer Commissur.

In den Seitensträngen Degeneration der Pyramidenbahn und Kleinhirnseitenstrangbahn, doch nur scbwach angedeutet; das übrige frei.

Im Vorderstrange keine Degeneration.

Bindegewebsepta und Centralcanal verbalten sich wio in den übrigen Theilen des Rückenmarkes.

Die Randzone ist intact, die hinteren Wurzeln führen zum Theil atrophische Nervenfasern.

Hinterhorn: In der spongiösen Zone der gelatinösen Substanz sind viele feine Nervenfasern erhalten, im eigentlichen gelatinösen Theile einige diesen durcbsetzende feine und grobe Faserbündel, die zur Substantia spon. giosa gehen. Letztere zeigt die wohlerhaltenen dicken Querschnitte der aufsteigenden Colonnen Clarke's und ein Netzwerk feiner markbaltiger Fasern, 
im hinteren Theile auch Querschnitte feiner longitudinaler Fasern. Einstrahlungsmassen aus den Hintersträngen sind nicht nachweisbar.

Die hintere Commissur ist ohne markhaltige Fasern, die vordere intact,

Von den grossen Ganglienzellen des Vorderbornes sind nur wonige vorhanden.

Das obere Cervicalmark - Grösse 10,0:7,7 Mm. - zeigt im Wesentlichen dasselbe Verbalten wie der Querschnitt der Halsanschwellung; in den Hirtersträngen insbesondere sind auch hier die vorderen seitlichen Theile und der hintere periphere Rand intact. In den Seitensträngen erscheint die Degeneration der Kleinhirnseitenstrangbahnen nicht sehr stark.

In der Medulla oblongata fällt schon makroskopisch die starke Verkleinerung auf; je weiter nach oben, umsomehr erscheint das Organ von vorn nach hinten zusammengedrücki. Ganz auffällig ist bei den Querschnitten durch Brücke und Medulla - bis zum Rückenmark bemerkbar - eine beträchliche seitliche, taillenartige Einkerbung (cf. Fig. I bei a) in der Gegend zwischen Vagus und Hypoglossus; dieselbe ist bedingt durch Volumenreduction des seitlichen Feldes der Substantia reticularis - die hier vorlaufenden Fasern kommen von der Brücke her -, ausserdem sind die in dieser Gegend gelegenen Seitenstrangkerne stark verkleinert, und es fehlen auch einige Fasern, die zur Olive führen.

Die Kerne der Goll'schen and mehr noch die der Burdach'schen Stränge, besonders der äussere Kern der Keilstränge sind stark verkleinert und arm an Zellen, - nicht nur degenerirt, sondern wesentlich, um ca. die Hälfte, kleiner als an normalen Präparaten.

Ganz besonders auffallend stark atrophisch ist im Keilstrange die äussere Abtheilung: hier fast faserlos, während sie normal einen starken Zapfen darstellt.

Auf der einen Seite zeigt sich das zwisehen aufsteigende Trigeminuswurzel und Keilstrang sich einsenkende Gebiet, welches nach Darkscbewitz als ein Ursprungsgebiet des Accessorius ${ }^{*}$ ) anzusehen ist, atrophisch. Atrophische Fasern finden sich auch im Accessoriusstamme dieser Seite.

Bedeutend ist die Atrophie im Seitenstrangkerne, der nur wenige erhaltene Zellen aufweist.

Ebenso sind die grossen Oliven stark atrophisch und ihr Gehalt an Zellen und markhaltigen Fasern deutlich verringert. Auch in der Längsausdehnung sind die grossen Oliven reducirt: wo das Corpus restiforme anfzutreten anfängt, zeigen sich an normalen Präparaten die Oliven gross, hier sind sie in dieser Gegend ganz klein geworden.

Das Stratum zonale erscheint arm an Fasern; von den Fibrae arcuatae externae sind keine markhaltigen Fasern wahrzunehmen. Vielmehr ist ein starker Defect hinter der die grossen Oliven umgebenden Sohicht bemerkbar;

*) L. Darkschewitz, Ueber den Ursprung and den contralen Verlauf des Nervus accessorius Willisii. Archiv f. Anat. und Physiol. 1885, anatom. Abtheil. S. 361. 
jedenfalls fehlt aber nur eines der dort verlaufenden Systeme; zu bedenken ist dabei allerdings, dass dies Bündel aus dem Kleinhirn kommend aussen herablaufen könnte.

In der Olivenzwischensebicht ist kaum ein Ausfall von Fasern zu constatiren, ebenso kaum verminderte markhaltige Fibrae arcuatae internae.

Die Pyramiden erscheinen gegenüber dem geringen Querschnitt der ganzen Medulla recht gross; ibre Grösse entspricht etwa der in einer normalen Medulla. In ihrem Gebiete ist kein Ausfall markhaltiger Fasern nach. weisbar: ihre Färbung ist an Weigert-Hämatoxylinpräparaten kaum etwas schwächer als die der Olivenzwischensehicht.

Deutlich ist die Degeneration der directen Kleinhirnseitenstrangbahn, in der sich nur wenige markhaltige Fasern nachweisen lassen.

Zwischen den sich kreuzenden Fasern der Fibrae arcuatae internae in der Raphe erscheinen einige perivasculäre Räume mit darin liegenden Gefässquerschnitten als ziemlich weite Spalten.

An einem Präparate aus dem unteren Theile der Medulla oblongata bomerkte ich etwa in der Mitte des Kernes der Burdach'schen Stränge eine Anhäufung auf dem Querschnitte getroffener markhaltiger Fasern deutlich ron der Umgebung sich abhebend; dieselben dürften vielleicht identisch sein mit dem im Burdach'schen Strange hier intact gebliebenen Theile, ron dem P. Flechsig annimmt, dass seine Fasern im Kerne der Keilstränge nach aussen verlaufen und sich beim Embryo frübzeitig mit Markscheiden umgeben.

Im f ypoglossuskern ist eine mässige Atrophie der Zellen wahrzunehmen, und die austretende Hypoglossuswurzel erscheint an markbaltigen Fasern verringert; das Marklager, welches den Hypoglossushauptkern vom Ventrikel trennt, ist wohlerhalten.

Das Corpus restiforme stellt sich arm an markhaltigen Nervenfasern und beträchtlich verkleinert - auf etwa $1 / 4$ reducirt - dar, so dass die aufsteigende Acusticus- und Trigeminuswurzel nur durch eine schmale Schicht vom peripheren Rande des Schnittes getrennt sind (cf. Fig. I. c. r.); der Kern des Strickkörpers ist besser erhalten als die Peripherie.

Die aufsteigende Trigeminuswurzel ist durchaus normal.

An höher gelegenen Schnitten in's Gebiet der Brücke übergehend, sieht man den Facialiskern otwas geschrumpft und in der austretenden Facialiswarzel einen Theil der Fasern entfärbt. Auf sämmtlichen vorhandenen Schnitten - die Reihe ist allerdings nicht lückenlos - ist der Facialis nur auf einer Seite anzutreffen; höchst auffällig ist dabei eine Theilung des Nerven in zwei Stränge, von denen der eine nach aussen abbiegend und lange im centralen Höblengrau verlaufend, sich zum Kleinhirn verfolgen lässt. Wahrscheinlich handelt es sich um einen abgesprengten Faserzug, der vom Kleinhirn herkommt und eine Strecke mit dem Facialis verlaufend sich dann in die Brückenfasern begiebt; möglicher Weise ist dieses Bündel auch als verlagerte Stria acustioa anzusehen.

In den Ursprungsgebieten des Acusticus ist nichts Abnormes nachzuweisen; die normal gelagerten Striae acusticae sind schwach entwickelt. 
Abducens-Kern und Wurzel sind normal; das Corpus trapezoid u m weist starkgefärbte Nervenfaserzüge auf.

Der sensorische Trigeminuskern ist wohlerbalten, man sieht die starken, gut gefärbten Faserzüge der sensorischen Wurzel (cf. Fig. II. V. s.) und ebenso den stark gefärbten von der Raphe kommenden Faserzug, der den gakreuzten Ursprung des Quintus darstellt; dagegen erseheint der motorische Kern beträchtlich kleiner und arm an Nervenzellen, dje zum Theil kleiner sind als die des sensorischen Kernes. Die mit der Thränensecretion in Zusammenhang gebrachten Zellen des oberen Trigeminuskerns zeigen ausser ihrer Verminderung an Zahl noch ein anderes sonst nicht beobachtetes Verhalten, indem die nach anssen gelegenen derselben mit schwarzem Pigment erfüllt sind.

In der Höhe des Trigeminusanstrittes ist die hochgradige Verkleinerung des ganzen Querschnittes selh auffällig; sowobl in der Breitendimension, besonders aber erscheint die Brücke im sagittalen Durchmesser verschmälert, so dass dieser nur $2 / 3$ von dem eines verglichenen normalen Präparates zeigt. Diese Verkleinerung betrifft nun hauptsächlich die vordere ventrale Abtheilung, so dass dieselbe an Quersohnitt die dorsale kaum wesentlich übertrifft. Diese Reduction des Querschnittes (und auch Längsschnittes) beruht auf einer hochgradigen Verminderung der markhaltigen Fasern in den Brückenschenkeln des Kleinhirns und einem fast vollständigen Fehlen des grossen Brückenganglions, welches nur wenige erhaltene Ganglienzellen und ganz wenig Netze aufweist. Gleichzeitig sind die aus der vorderen Brückenabtheilung in der Raphe dorsalwärts ziehenden Faserzüge fast rollständig geschwunden. Querfasern in der Brücke sind noch rorbanden (cf. Fig. II.).

Sehr schön erhalten ist der obere Centralkern.

In der Mitte der Brücke erscheint die Schleife ziemlich gut tingirt (cf. Fig. II.); das Bindegewebe etwas mehr entwickelt als normal and die Markfasern der Schleife (laterale Schleife), die um den Bindearm laufend, in das vordere Marksegel gehen, weisen nur eine geringe Atrophio auf, die viel. leicht nur als einfache Verdünnung anzusprechen ist. Die stark pigmentirten Ganglienzellen des Locus coeruleus treten deutlich hervor; die Faserbündel der absteigonden Trigeminuswurzol sind gut gefärbt; der Querschnitt des hinteren Längsbündels ist ziemlich beträchtlich und hebt sich gut von der helleren Umgebung ab.

Die oentrale $H a u b e n b a h n$ tritt deutlich hervor, indem sie sich stark gefärbt vou der hellen Umgebung abgrenzt; das Feld dahinter ist hier atrophisch, wohl vorhanden, aber schlecht entwickelt.

Im ventralen Theile erscheinen die stark tingirten Quersehnitte der Py ramiden von wenigen markhaltigen Fasern umgeben (cf. Fig. II. f.)

Der Querschnitt des Bindearmes ist relativ pergrössert, die markhaltigen Nervenfasern desselben mit Weigert's Hämatoxylin tief blau gefärbt (cf. Fig. II. Brej.).

In der hinteren Vierhügelgegend zeigen sich die hinteren Längsbündel, Schleifenschicht, die sich kreuzenden Fasern der Bindearme stark gefärbt, der Trochleariskern woblerhalten. 
In der vorderen Vierbügelgegend erscheint der vordere Vierhügel selbst sehr abgeflacht, das Corpus geniculatum mediale gar nicht vorragend und die Thalami optici beider Seiten näher an einander gerückt als normal.

Bindearme und hintere Längsbündel haben normale Färbung. Die im Zusammenhange mit der Bräcke stebende Substantia nigra Sömmeringii ist zwar gut gefärbt, aber dentlich verschmälert und enthält äussert wenig pigmentirte Zellen.

Der Hirnschenkelfuss ist um ein Drittel verkleinert.

An Schnitten aus der Mitte des vorderen Vierbügels erscheint der normal gefärbte rothe Kern der Haube, wohlerbaltene Fasern des Nervus oculomotorius und das mächtige Bündel des Tractus opticus, der in das Corpus geniculatum laterale eintritt. Der Luys'sche Körper erscheint beträchtlich abgeplattet und verkleinert.

Schnitte durch den vorderen Rand des vorderen Vierhügels zeigen deutlich gefärbt die Fasern, die vom Vierhügel zum Thalamus opticus führen, die Bündel, die vom lateralen Rande des rothen Kernes ausstrahlen, die mächtigen Züge der hinteren Commissur und deren ventrale Fasern, die um den Aquaeductus Sylvii za den noch erkennbaren hinteren Längsbündeln verlaufen. Nirgends ist hier ein Ausfall markhaltiger Fasern erkennbar.

Der vierte Ventrikel ist ungewöbnlich gross im Verhäliniss zu dem verringerten Querschnitte; seine Gestalt ist breit gozogen, maulförmig, an den Seiten mit tiefen Einsenkungen (cf. Fig. I. und II. V. IV.); ebenso ist das centrale Höhlengrau, in dem sehr schöne Zellen erhalten sind, relativ sehr entwickelt, und der Aquaeductus Sylvii ist weiter als an normalen Präparaten.

Das Kleinbirn (cf. Fig III.) zeigte schon makroskopisch ebenfalls eine hochgradige Verkleinerung; genaue Masse desselben fehlen, da ich dasselbe nicht mehr in toto vorfand.

Die Blätter erscheinen als schlaffe schmale Lamellen, die weite Zwischenräume zwischen sich haben; eine bessere Consistenz zeigen nur Mandel, Wurm und Flocke, deren Lamellen auch makroskopisch auf dem Querschnitte breiter, dichter gelagert und stärker gefärbt sich darstellen; ebenso ist schon makroskopisch ein besser erbaltener Zustand erkennbar an den einander zugekehrten Läppchen, die der Vorderfläche des Lob. semilanar. post. und der hinteren Fläche des Lob. lunat. post. angehören.

Die mikroskopische Untersuchung einer Reihe von Schnitten; deren Richtung in die Transversalebene fällt, führt zu den nacbstehenden Resultaten.

Die Zellen des Nucleus dentatus stehen zienilich dicht gedrängt, sind stark gefärbt - nach Weigert -, zum Theil geschrumpft. Dachkern, Kngelkern und Pfropf zeigen nicbts Abnormes.

In der Markfaserschicht erscheint der Markkern des Corpus dentatum nur in seiner ventralen Partie mit gut gefärbten Fasern; das das Corpus dentatum umgebende Vliess dagegen arm an markhaltigen Fasern; noch stärker ist der atrophische Zustand in den Faserbündeln der einzelnen Läppchen; an diesen atrophischen Stellen bemerkt man viele ungefärbte 
Bindegewebsfasern zwischen den vereinzelt vorhandenen markhaltigen Nervenfasern.

In der Rinde erscheint die Körnerschicht gegenüber normalen Präparaten etwas verschmälert und ärmer an Körnern; die moleculäre Schicht ist ebenfalls verschmälert. Am auffälligsten ist der Ausfall der grossen Purkinje'schen Zellen, dagegen sind die horizontalen Plexus in der Kleinhirnrinde sehr wohl erhalten, auch da, wo die Purkinje'schen Zellen völlig fehlen.

Wo die Zellen fehlen, ist ihr Ausfallen ein vollständiges, nirgends trifft man Spuren degerierirter Zellen.

Ueberall, wo dio Markleisten besser erhalten sind, zeigen sich auch deutlich zablreichere Zellen; stellenweise finden sich Läppohen ohne jede Spur von Zellen und Markfaseri.

Im Allgemeinen erscheinen dio oberen Partien des Kleinhirns von der Atrophie viel mehr betroffen als die unteren; im Einzelnen lässt sich folgender Befund feststellen.

In Wurme (cf. Fig. I. Vr. i.) finden sich verbältnissmässig wenig Defecte markhaltiger Fasern; auch die Purkinje'schen Zellen sind noch ziemlich reichlich vorhanden, wenn auch sichtlich vermindert. Wabrscheinlich gehen viele der orhaltenen Fasern nicht zu den Purkinje'schen Zellen sondern zu den Plexus, die mit jenen Zellen nicht in Zusammenhang stehen. Die vorderen Theile des Wurmes sind etwas weniger gut erhalten als die hinteren, doch ist der Unterschiod nicht beträchtlich.

In gutem Zustande befinden sich Tonsille, Uvula und Nodulus, deren Rinde zablreiche Purkinje'sche Zellen, deren Markschicht wohlgefärbte Fasern aufweisen.

Eine wohlerhaltene, wie erwähnt schon makroskopisch sich deutlich abhehonde Partie stellon auch die oinander zugewandten, der Vorderflächo des Lob. super. poster. s. semilunaris und der Hinterfläche des Lob. lunat. poster. angebörenden Läppchen dar (cf. Fig. III).

$\mathrm{Ab}$ und $\mathrm{zu}$ finden sich $\mathrm{im}$ Lob. cuneiformis gute Partien, doch zeigt sich hier keine gleichmässige Vertheilung des Guterbaltenen, nur scheinen im Allgemeinen die vorderen Theile des Lob. cuneiformis, die nach der Flocke zu liegen, besser erhalten.

In den übrigen Theilen trifft man nur ganz vereinzelt hier und da auf Purkinje'sche Zellen, und zwar findet man an solchen Stellen meist mehrero derselben zasammengedrängt.

Bei der Vergleichung der vorliegenden Kleinhirnschnitte mit fötalen Präparaten orgiebt sich, dass an urserem Kleinhirn diejenigen Thoile am besten erhalten sind, welcho besonders frübeitig (Wurm) oder zu allerletzt ihre Marksobeiden entwickeln (Mandel, Gebiet des Lob. semilunar. post. und Lob. lunat. post.), während die bezüglich ihrer Entwicklungszeit in der Mitte liegenden am schlechtesten ausgebildet sind; letztere sind gleichzeitig die Theile, die sich durch das Vorbandensein von Bogenfasern auszeichnen. 
Es liessen sich einige Beobachtungen betreffs der Verbindungen des Kleinhirns mit anderen Gehirntheilen machen.

So scheinen aus den zulelzt erwähnten schlecht ausgobildeten Partien gerade hauptsächlich die Fasern zu kommen, welche in dio grosseen Oliven gehen; ausserdem zejgte sich auch die Zahl der aus dem sebr rducirten Corpus restiforme in die Oliven einstrahlenden Fasern sehr gering.

Im Wurm tritt eine Anzahl guterhaltener Fasern auf, die aus dem erhaltenen Theile des Corpus restiforme stammen.

Deutlich zu verfolgen ist, wie markhatige Fasern von den Kleinhirnseitenstrangbahnen her in die Markfaserbündel des Kleinhirns eintreten.

Für die Bindearme ist zu beobachten, dass sich dieselben relativ wenig in die hinteren und oberen Partien der Kleinhirnhemisphären verzweigen, sondern vorzugsweise nach den basalen Theilen gehen, und dass dieselben nach der Mitte des Kleinhirns zu ausstrahlen, nicht sich nach aussen wenden; in ihrem Verlauf deutlich zu verfolgen sind einige Bündel des Bindearmes, die sich in die hier wohlerhaltene Gegend des Lob. lunat. post. und Lob. semilun. post, fortsetzen und dort Fasern abgeben. -

Die Pi a mater haftet den nervösen Centralorganen ziemlich fest an, besonders im anteren Dorsal- und im Lendenmark, doch so, dass sie ohne Substanzverlust abgelöst werden kann.

Die im Vorstehenden dargestellten Resultate stellen sich, soweit sie sich auf das Rückenmark beziehen, den oben angefübrten Sectionsbefunden, die Fälle Friedreich'scher Tabes betrafen, gleichlautend zur Seite; anderseits aber enthalten sie einige Ergebnisse, welche in keinem der früheren Fälle zur Beobachtung gelangt waren: die Kleinhirnaffection und die Veränderungen in Medulla oblongata und Pons; allerdings ist von Hammond (cf. No. 41 des Lit.-Verzeichnisses) ein Causalnexus dieser mit der vorliegenden Krankheit angenommen werden.

Die Zahl der in der Literatur bekannten Fälle von Cerebellaratrophie ist nicht sehr gross - am Schlusse folgt eine Zusammenstellung derselben - Alle diese Fälle weisen sehr viel Uebereinstimmendes auf: überall zeigt der atrophische Process das nämliche pathologisch-anatomische Verhalten: Schwund der Rindenschicht und Ausfall der Purkinje'schen Zellen sowie Bindegewebswucherung in der Markschicht; ebenso constant erscheinen als Folge der Kleinhirnatrophie Notilitätsstörungen - (von Huppert (cf. No. 120), Kohts (Virchow's Archiv, Bd. LXVII S. 425), Schulze (cf. No. 134), Fraser (No. 124), Seppilli (No. 123), Kirchhoff (No. 127), Claus (No. 125), Ferrier (No. 133) u. A. beobacbtet) -, Anomalien der Augenbewegungen - (Ferrier, Schulze, Budge, Wegner) - 
Sprachstörungen - (Huppert, Schulze, Fraser, Sepilli, Kirchhoff, Sommer (cf. No. 131). -

Kirchboff bemerkte die Aehnlichkeit atrophischer Kleinhirnparthien mit gewissen Stadien der embryonalen Entwicklung desselben und kam zu dem hypothetischen Resultate, dass auf entwicklungsgeschichtlicher Basis entstandene Kleinhirnatrophie mit folgender Sclerose zu Motilitätsstörungen führen kann (cf. No. 127).

Nun ist die Frage zu entscheiden, wodurch im vorliegenden Falle die Ataxie bedingt ist. Es ergiebt sich, dass alle Gebiete des Centralorganes, in denen Centren für die Coordination der Bewegnngen gelegen sind: Kleinhirn, Corpora quadrigemina, Pons und Kleinhirnschenkel*) von dem Krankheitsprocesse ergriffen sind, und dass auch die Bahnen, die von der Peripherie her zu denselben leiten, insbesondere die von $\mathrm{Charcot}$ als anatomisches Substrat der Bewegungsataxie bezeichneten Zones radiculaires postérieures von demselben betroffen sind; a priori wird also kein einzelner dieser Factoren für die resultirende Ataxie verantwortlich zu machen sein. Setzen wir aber eine Entwicklungshemmung sowohl des Kleinhirns wie der Medulla oblongata und spinalis und verlegen wir diese in die Zeit des 7. bis 8. embryonalen Monates, so haben wir damit die Erklärung für ein dem Entwicklungsgrade dieser Zeit entsprechendes Cerebellum, dessen Weiterbildung unterbrochen wurde, als die Entwicklnng der Purkinje'schen Zellen eben erst zum Theil begonnen hatte, und anderseits für ein Rückenmark, dessen Fasersysteme auf einer bestimmten Entwicklungsstufe stehen blieben und für spätere sclerotische Processe prädisponirt waren (Friedreich).

Im Sinne der Annahme ejner congenitalen Abnormität einzelner Gehirnparthien ist, abgeseben von der hereditären Belastung auch die auffällige relative Grösse des vierten Ventrikels und des centralen Höhlengrau herbeizuziehen.

Die Atrophie des Kleinhirns erklärt zunächst die Atrophie der Oliven: durch von Gudden's Experimente, die Marchi**) bestätigt hat, ist Atrophie der Oliven bei Kleinhirnexstirpation nachgewiesen; derselbe zeigte, dass bei der gleichen Operation auch das Corpus restiforme und die Kleinhirnseitenstrangbahn der operirten Seite atrophiren; so könnte in unserem Falle die theilweise Degeneration dieser beiden Gebilde von der Cerebellaraffection abhängig

*) Friedreich. Virchow's Archip Bd. 68. S. 208.

**) cf. Gudden, Neurol. Centralbl. 1882. S. 455. - Marchi, Rivista sperimentale di fren. 1888. XIII. p. 446.

Arehiv f. Psychiatrie. XXII, 1. Heft. 
sein, anderseits wird aber dieselbe auch durch den aufsteigenden degenerativen Process im Rückenmarke erklärt, sodass gewissermassen die vom Kleinhirn und von dem Rückenmark ausgehenden Linflüsse sich in der Medulla oblongata entgegengearbeitet haben, und als Begründung für diese Anschauung lässt sich wohl der Umstand herbeiziehen, dass im obersten Cervicalmarke die Veränderung der Kleinhirnseitenstrangbahnen weit weniger bemerkbar ist als in den oberund unterhalb davon gelegenen Segmenten. Oder aber, es könnte die Annahme noch näher liegen, dass die durch die Kleinhirnatrophie geschwächten Corpora restiformia und Kleinhirnseitenstrangbahnen der zerstörenden Wirkung des vom Spinalmark aufsteigenden Processes um so leichter erlagen.

Nicht absolut unmöglich, aber durch keine sicheren Beweise gestützt, wäre es, dass die hier gleichmässiger als bei Tabes dorsalis auftretende Hinterstrangdegeneration secundär absteigend das Resultat der Kleinhirnatrophie sei: dass in Folge der Kleinhirnatrophie die Kerne der Hinterstränge degenerirten, und dass die degenerirten Zellen der Kerne mit den geschrumpften Fasern der Hinterstränge zusammenhängen, während die nicht degenerirten Hinterstrangsfasern durch die erhaltenen Zellen der Kerne zum Grosshirn verliefen.

Die Ansicht nun, als ob die Veränderungen in Kleinhirn, Brücke und verlängertem Marke Secundärerscheinungen im Anschlusse an die Rückenmarkserkrankung seien, erachte ich durchaus für nicht statthaft; es finden sich in den genannten Organen keineswegs die Zeichen einer regressiven Jetamorphose, vielmehr imponiren dieselben als von vornherein mangelhaft ausgebildet: so finden wir im Kleinhirn keinerlei Spuren geschrumpfter Elemente, sondern dieselben erscheinen nur kleiner und weniger zahlreich als normal, es gelingt zum Beispiel an den Stellen, wo die Purkinje'schen Zellen feblen, absolut nicht, auch nur die geringsten Residuen ron ursprünglich daselbst vorbanden gewesènen Zellen zu entdecken. Das scheint mir binreichend für eine primitive Erkrankung des Organes zu sprechen; was einige im Marke des Kleinhirns anzutreffende Fasern betrifft, welche das Zeichen einer wirklichen Degeneration tragen, so ist deren Alteration, ohne der obigen Ansicht zu nahe zu treten, recht wohl durch die später eingetretene aufsteigende Degeneration zu erklären.

Ebenso ist daraus, dass die Kerne der Goll'schen und Burdach'schen Stränge nicht nur atrophisch sondern um die HäIfte kleiner als normal sind, anzunehmen, dass sie schon congenital geringer an Volumen waren, und dass diese Grössenverminderung 
nicht lediglich als eine Folge der aufsteigenden Hinterstrangsdegeneration aufufassen ist.

Danach ist nun sofort plausibel, dass in den ersten Jahren für den Patienten seine mangelhaft ausgebildeten Centralorgane, in die wir die Coordinationscentren verlegen, ihre physiologischen Functionen noch völlig normal verrichten konnten, so lange die zuleitenden Bahnen im Rückenmarke noch intact waren; es ist ja auch durch das Experiment nachgewiesen, dass Kleinhirnläsionen, die nur einen Theil desselben betreffen, ganz symptomlos verlaufen können; eine Aenderung trat erst dann ein, als sich zu jener ursprünglichen Affection die Erkrankung der centripetalen Bahnen gesellte, und je weitere Fortschritte dieser aufsteigende Process machte, um so intensiver traten auch die in Frage kommenden Erscheinungen zu Tage, wie aus der Krankengesehichte hervorgeht.

Darum erscheint mir die von den Hintersträngen, welche primitiv erkrankten, ausgehende Rückenmarksaffection - sei es, dass deren Propagation auf die anderen Rückenmarksabschnitte durch directe transversale Verbreitung auf graue Substanz und Seitenstränge oder durch Ausbreitung auf die Seitenstränge unter Vermittelung einer durch die Hinterstrangsdegeneration angeregten Leptomeningitis spinalis chronica*) erfolgte, - als die hauptsächlichste Ursache der vorliegenden Coordinationsstörungen anzusprechen zu sein, durch welche das an sich immerhin noch lejstungsfähige Kleinhirn ausser Thätigkeit gesetzt wurde.

Sind danach im Allgemeinen die Erscheinungen der Ataxie, die der Kranke darbot, hinsichtlich ihrer Veranlassung klargelegt, so erübrigt es noch, mit einigen Worten anf einzelne besondere Symptome einzugehen.

Die Sprachstörungen zunächst, anfangs in Schwerfälligkeit bestehend und sich später immermehr steigernd, kamen nach mehrjährigem Bestande der Krankheit in fast allen Fällen der Friedreich'schen Tabes zar Beobachtung und wurden anderseits auch meist bei Kleinhirnatrophie als hervorragendes Symptom angegeben; so beschreibt dieselben Huppert (cf. No. 120 des Lit. Verz.) in einer unserem Falle ganz analogen Weise; sie lassen sich recht wohl einfach als eine Theilerscheinung der allgemeinen motorischen Ataxie auffassen, indess ist im vorliegenden Falle auch eine Alteration im Ursprungsgebiete des Hypoglossus und in der austretenden Warzel desselben deutlich erkennbar.

*) Friedreich, Virchow's Archiv Bd. 70. S. 150. 
Von Seiten der Augen lagen hier keine Abweichungen von der Norm vor, mit Ausnahme der später zu besprechenden Mitbewegungen; es lassen sich auch im Gebiete der in Frage kommenden Nerven, Opticus, Oculomotorius, Trochlearis, Abducens, keinerlei Veränderungen nachweisen.

Bemerkt sei an dieser Stelle, dass im oberen Trigeminuskerne nur wenige und degenerirte Zellen gefunden wurden; beim Nachfragen ergab sich, dass der Kranke in den letzten Jahren trotz häufigen Niedergeschlagenseins und gedrückter Stimmung nicht im Stande gewesen ist zu weinen, ein Umstand, der möglicher Weise für den behaupteten Zusammenhang jener Zellen ("Weinzellen") mit der Thränensecretion gedeutet werden kann.

Sehr auffällig waren bei dem Kranken die krampfhaften Mitbewegungen im Gesicht beim Gehen und Lesen, die sich steigerten, je länger derselbe intendirte Bewegungen vornahm, Bei der Bedeutung der erkrankten Partien des Centralorganes für harmonische Ausführung der Bewegungen erscheinen dieselben ohne Weiteres erklärlich; und der Umstand, dass die Erscheinungen sich steigerten, je zahlreichere Impulse Patient verwendete, dagegen geringer wurden, wenn er sich in einer lage befand, die möglichst wenig motorische Impulse erforderte, zeigt, dass sie als die Folgen der Schwäche und physiologischen Insufficienz jener Gehirntheile anzusprechen sind. Ausserdem finden sich aber auch die die betreffenden Gesichtspartien versorgenden motorischen Nerven afficirt.

Es wurden beobachtet Aufreissen der Augen, Vertiefung der Nasolabialfalte und Contraction der Kinn- und Mundmuskeln, hierfür ist der Facialis verantwortlich zu machen; später traten weites Aufreissen des Mandes und Kaubewegungen beim Sprechen hinzu; die dabei betheiligten Muskeln fallen in das Gebiet der motorischen Trigeminusfasern; es zeigten nun der Facialiskern wie der motorische Quintuskern geschrumpfte Kerne und ihre austretenden Wurzeln degenerirte Fasern.

Ausserdem bot Patient Rechtsdrehen des Kopfes and der Augen bei intendirten Bewegungen dar, z. B. beim Lesen im Bette, im weiteren Verlaufe dieselbe Erscheinung beim Gehen; hierfür ist möglicher Weise die oben erwähnte Atrophie in dem von Darkschewitz nachgewiesenen Accessoriusursprungsgebiete zwischen aufsteigender Trigeminuswurzel und Keilstrang ${ }^{*}$ ) verantwortlich zu

*) Arohir f. Anat. u. Physiol. 1885, anat. Abtheil. S. 361. 
machen, indem in den hier entspringenden Accessoriusfasern eine Bahn für die Kopfhaltung zu suchen wäre.

Für den auffälligen Lmstand, dass die Ataxie auf der rechten Seite stärker, die rohe Kraft daselbst mehr vermindert war und zum Scblusse im rechten Arme sich choreartige Zuckungen bemerkbar machten, lässt sich eine anatomische Ursache nicht nachweisen, da im Uebrigen in keinem der untersuchten Theile eine einseitig mehr hervortretende krankhafte Veränderung deutlich erkennbar ist.

Von den übrigen Symptomen, die der motorischen Sphäre angehören, sind diejenigen zu bemerken, welche von den grossen Ganglienzellen des Vorderhornes und von den Pyramidenbahnen abhängig sind. Wir finden denn auch in Folge der allmälig entwickelten Atrophie eines Theiles der Vorderhornzellen eine theilweise Atrophie der vorderen Wurzeln und das Auftreten von Schwäche und Atrophie in den Muskeln, noch nicht aber Lähmung, da ein Theil der Ganglienzellen noch intact ist. Die Erscheinungen der Muskelatrophie traten nicht sehr in den Vordergrund, da die Seitenstrangaffection jedenfalls erst spät hinzutrat, wie sie sich auch auf den Querschnitten weit weniger ausgeprägt zeigt; daher treten anch die abnormen Spannungen in den Muskeln und die Contracturen einzelner Glieder erst spät in dem Krankheitsbilde auf.

Bemerkenswerth und abweichend vom gewöhnlichen Verlaufe ist das Erhaltenbleiben, zeitweise sogar gesteigerte Verhalten des Patellarreflexes, trotz der Veränderungen in den Keilsträngen, durch welche die tiefen Reflexe ziehen; man kann hier wohl annehmen, dass der Weg derselben gerade die Theile jener Stränge berührt, welche in unserem Falle von der Degeneration verschont blieben, und die theilweise Steigerung dürfte die Folge der Seitenstrangerkrankung oder einer erhöhten Reizbarkeit der grauen Substanz sein.

Von sensiblen Erscheinungen finden sich in der Krankengeschichte im Beginn einmal Kreuzschmerzen, dann das Gefübl des Geschwollensejns in Nacken, Schulter und Hinterkopf verzeichnet; diese sind theils durch Betheiligung der Rückenmarkshäute, theils durch Reizung der erhaltenen sensiblen Fasern durch den Degenerationsprocess der übrigen zu erklären.

Von eigentlichen Störungen der Sensibilität ist aber nicht die Rede; die Erhaltung derselben bei fast völliger Degeneration der Hinterstränge und ausgebreiteter Affection der hinteren Wurzeln, wie sie auch von Friedreich (cf. No. 2, 11, 12), Prevost (cf. No. 15), Schultze (cf. No. 25, 39, 51), Erlicki (cf. No. 65, 80) beobachtet wurde, legt die Anschaung nahe, dass die Bahnen für Schmerzgefühl 
und Tastsinn nicht in den Hintersträngen verlaufen, und macht die Ansicht Schiff's wahrscheinlich*), dass diese Leitung lediglich durch die graue Substanz vermittelt wird, wenn wir nicht annehmen wollen, dass diese Bahnen gerade die Gebiete durchlaufen, welche in den Hintersträngen hier von der Krankheit verschont geblieben sind; als solche kommen die Lissauer'sche Randzone und das Feld zwischen hinterer Commissur und Hinterhorn in Betracht. Das letztere zeigt sich bei der gewöhnlichen Tabes constant intact, während bei dieser Krankheit die Sensibilitätsstörungen zu den charakteristischsten Symptomen gebören; aus diesem Grunde kann dieses Feld nicht in Frage kommen; anders verhält es sich mit der Lissauer'schen Randzone, welche bei gewöhnlicher Tabes ergriffen, bei der Friedreich'schen Ataxie dagegen immun erscheint; und dieser Umstand kann in Verbindung mit der Sensibilitätsstörung bei Tabes und dem Mangel dieses Symptomes bei der hereditären Ataxie zur Vermuthung eines Zusammenhanges zwischen Randzone und Sensibilitätsleitung führen. So wäre es möglich, dass unbeschadet der obenangeführten Meinung von Schiff die Leitungsbahnen für die Sensibilität (Schmerzgefühl und Tastsinn) in denjenigen feinen Fasern zu suchen sind, welche nach Lissauer's Untersuchungen (cf. No. 84) aus den hinteren Wurzeln kommend zwischen Hinter- und Seitensträngen eben die genannte Randzone bilden, zunächst eine Phase longitudinalen Verlaufes durchmachen und, im Einzelnen verschieden lang, schliesslich alle in die graue Substanz eintreten und also, ohne die Hinterstränge zu passiren, centralwärts verlaufen.

Directe Beweise für diese Annahme fehlen allerdings.

In der mehrfach erwähnten Abhandlung macht Lissauer noch auf einen Punkt aufmerksam, welcher in Folge dessen bei der vorliegenden Untersuchung eine besondere Sorgsamkeit erfuhr; er fübrt an, dass möglicher Weise bei der Friedreich'schen Tabes trotz der Atrophie der Ganglienzellen der Clarke'schen Säulen die Einstrahlungsfasern in dieselben aus den Hintersträngen verschont blieben, und dass aus dem Verhalten der feinen markhaltigen Fasern zwischen den Zellen vielleicht Aufschluss über die Bedeutung derselben erhalten werden könne. Diese Hoffnung erwies sich bei unserem Falle aber leider als vergeblich; denn wie schon oben bei der Beschreibung der mikroskopischen Befunde ausgeführt wurde, waren an den untersuchten Querschnitten weder Einstrahlungsfasern aus den Hintersträngen in die Clarke'schen Säulen, noch Fasern aus dem Vorder-

*) Lehrbuch der Physiologie des Nervensystems 1858-59. 
horn, die zu denselben verlaufen, noch die Flechsig'schen Kleinhirnbündel nachzuweisen, und ebensowenig war eine Verbindung der feinen Markfasern des die Zellen der Clarke'schen Säulen umgebenden zarten Netzwerkes mit jenen Zellen irgendwo erkennbar.

Wir können nun zum Schlusse die gefundenen Resultate dahin zusammenfassen, dass dem geșchilderten Krankbeitsbilde ein complicirter anatomischer Process zu Grunde liegt, der sich zusammensetzt aus einer auf Entwicklungshemmung beruhenden combinirten Systemerkrankung, wie eine solche als Ursache der hereditären Ataxie angenommen wird, und einer daneben bestehenden mangelbaften Ausbildung von Kleinhirn, Brücke und verlängertem Marke - es sei hierbei noch auf deren gemeinsame Abstammung ans dem dritten Hirnbläschen hingewiesen. - Der erstere Factor ist als der wichtigere anzusehen: der fortschreitende Process im Rückenmarke hatte die langsame aber stetige Weiterausbreitnng der Krankheitserscheinungen zur unmittelbaren Folge, wobei der Cerebellaraffection die Rolle eines unterstützenden Momentes zukam.

\section{Literatur über hereditäre Ataxie*).}

1. Carré, De l'ataxie locomotrice progressive. Thèse de Paris 1862.

2. Friedreich, Ueber degenerative Atrophie der spinalen Keilstränge. Virohow's Archiv Bd. 26. 27.

3. Topinard, De l'ataxie locomotrice. Paris 1864.

4. Carré, Nouv. recherches sur l'ataxie locomotrice. 1865.

5. Bradbury, Brit. Med. Journal 1871. p. 565.

6. Pierret, Arch. de Physiol. Vol. IV. 1871-72.

7. Charpenter, Report of Med. Society of London. Lancet 1871. p. 779.

8. Bouchut, Ataxie locom. et sclérose des cordons post. de la moëlle chez les enfants. Gaz. des Hôp. 1874. No. 38. p. 297.

9. Kellogg, Two cases of locomotor Ataxia in children. Arch. of Electrol. and Neurol. Vol. II. 1875 . p. 182.

10. Hitzig, Berliner klin. Wochenschr. 1875. S. 513.

11. Friedreich, Ueber statische Ataxie und atactischen Nystagmus. Dieses Archiv 1875. Bd. VII. S. 235.

12. Friedreich, Ueber Ataxie mit besonderer Berücksichtigung der hereditären Formen. Virchow's Archiq Bd. 68. S. 145, Bd. 70. S. 140.

*) Ein Theil dieser Angaben findet sich zusammengestellt in Brain Vol. IX. 1886. p. 145 von Judson S. Bury. 
13. Dresohfeld, Family praedisposition in locomotor Ataxy. Manchester and Liverpool Hospital-Reports. Vol. IV. 1876. p. 93.

14. Shaw, A case of Ataxia in a Child. Tr. Am. Neurol. Assoc. N. Y. 1877. II. p. 85.

15. Prévost, Ataxie locomotrice. Sclérose des cordons postérieurs compliquée d'une sclérose symmetrique des cordons latéraux. Arch. de Physiol. norm. et pathol. 1877. p. 764 .

16. Vulpian, Maladies du système Nerr. 1877. p. 245.

17. Kahler und Pick, Ueber combinirte Systemerkrankungen des Rückenmarkes. Dieses Archiy Bd. VIII. 1877.

18. Erb, Krankheiten des Rückenmarkes. Handb. d. spec. Pathologie und Therapie von Ziemssen. Bd. XI. 1878.

19. Eulenburg, Lehrbuch der Nervenkrankheiten. Bd. II. 1878. p. 458.

20. Westphal, Ueber combinirte Erkrankung der Rückenmarksstränge. Dieses Archiv Bd. VIII. und IX. 1877 and 1878.

21. Reymond, Areb. de Physiol. X.

22. Möbius, Ueber die hereditären Nerrenkrankheiten. Samml. klin. Vorträge v. Volkmann 171. 1879.

23. Seeligmüller, Centralbl. für Nerrenheilkunde, Psychiatrie ete. Bd. II. 1879. S. 468. Bd. III. 1880. S. 425.

24. Rabusin, Virohow's Archir Bd. 76. S. 74. 1879.

25. Fr. Schultze, Uөber combinirte Strangdegenerationen in der Medulla spinalis. Virchow's Archiv Bd. 79. S. 132. 1880.

26. Seeligmüller, Hereditäre Ataxie mit Nystagmus. Dieses Archiy Bd.X. 1879. S. 222.

27. Se elig müller, Sclerose der Hinterstränge. Handbuch der Kinderkrankheiten von Gerbardt. 1880 . S. 187.

28. Kahler und Pick, Neuer Fall von gleichzeitiger Erkrankung der Hinter- und Seitenstränge. Dieses Archiv Bd. X. S. 179. 1879.

29. Henoch, Atactische Symptome durch Genitalreizung bei einem 7 jährigen Knaben. Wiener med. Blätter 1880. No. 12. S. 292.

30. Schmid, Ueber hereditäre Ataxie. Correspondenzblatt für Schweizer Aorzte. Bd. IV. 1880 . S. 97.

31. Gow ers, A. family affected with locomotor Ataxia. Transactions of the clin. society. London 1880 . Vol. XIV. p. 1.

32. Hollis, Locomotor Ataxy in a Boy. Brit. med. Journal. 1880. II. 167.

33. Strümpell, Ueber combinirte Systemerkrankungen im Rückenmarke. Dieses Archiv Bd. XI. S. 27. 1880.

34. Sioli, Dieses Archiv Bd. XI. 1880.

35. Grasset; Traité pratique des maladies du système nerveux. Paris 1881. p. 321.

36. Brousse, De l'ataxie héréditairo. Paris 1882; oitirt Mendel's Centralbl. 1882 . S. 527.

37. Féré, Ataxie héréditaire. Maladie de Friedreich. Solérose diffuse de 
la moëlle et du bulbe. Progr. med. X, 1882, p. 890; citirt Mendel's Centralblbl. 1882. S. 558.

38. Daridson, Two cases of Ataxia in obildren. Med. Times and Gazette. April 22. 1882.

39. Schultze, Beitrag zur Lehre ron der seg. Deg. im Rückenmarke des Menschen. Dieses Archiv XIV. S. 359. 1883.

40. Anr. Bianchi, La malattia del Friedreich. Gazzette degli Ospitali. 1882. No. 95.

41. Hammond, On the so-called family or bexeditary form of locomotor Ataxy. Journ. of Nerv. and Ment. disease. Vol. IX. 1882. July. No. 3. p. 484 ; cit. Mendel's Centralbl. 1882. S. 557.

42. Leubuscher, Ein Fall von Tabes dorsalis im frübesten Kindesalter. Berliner klin. Wochenschr. 1882. No. 39. S. 590.

43. D'Arey Power, A case of hereditary locomotor Ataxy. St. Bartholomews Hosp. Reports 1882. XVIII. p. 305.

44. W. (F.), Diffuse sclerosis of the spinal cord. and medulla oblongata. Disease of Friedreioh. Phil. M. Times 1882-83. XIII. p. 372.

45. Brit. Med. Journal 1882. I. p. 627.

46. Erlen meyer, Zur Lehre von den Coordinationsstörungen in Kindesalter. Centralbl. f. Nervenkrankh. 1883. No. 17. p. 385.

47. Leyden, Tabes dorsualis. Realencyclopädie der ges. Heilkunde, herausgeg. von Pr. Eulenburg. Wien 1883. Citirt Mendel's Centralblatt 1883 .

48. Rätimeyer, Ueber hereditäre Ataxie. Virchow's Archiv Bd. 91. 1883. S. 106.

49. StadeImann, Doutsches Archiv f. klin. Med. XXXIII. II. S. $125,1883$.

50. Sepilli, Atassia ereditaria, o malattia di Friedreich. Rivista sperim. di Freniatria 1883. p. 335.

51. Schultze, Wanderversammlung der Südwestdeutschen Neurologen und Irrenärzte in Baden am 16. und 17. Juni 1883. Dieses Archiv Bd. XV.

52. Jacubowitseh, Tabes dorsalis im Kindesalter. Archir f. Kinderbeilkunde. 1883 . XII. S. 187.

53. Massalongo, Della Malattia del Friedreich. Rivista Veneta di scienza mediche 1884 .

54. Musso, Sulla malattia del Friedreich, atassia locomotrice oreditaria. Rivista Clinica 1884. Cit. Mendel's Centralbl. 1885. S. 11.

55. Teissier, Ataxie héréditaire avec dégénérescenoe des faisceaux postér. de la moëlle. Lyon méd. No. 19. 1884.

56. Rosentbal, Diagnostik und Therapie der Rückenmarkskrankheiten. Wien und Leipzig 1884.

57. Longuet, Critical digest of Friedreich's disease. Union med. 18. Mai 1884 .

58. Déjérine, Arch. de Physiol. 1884.

59. Wälle, $Z_{w \in i}$ neue Fälle von bereditärer Ataxie. Correspondenzblatt für 
Schwoizer Aerzte No. 2. 1884 . Cit. Centralblatt f. d. med. Wissensch. 1884. S. 304 .

60. Ormerod, On the so-called hereditary Ataxia, first described by Friedreich. A critrical digest. Brain, Vol. VII. p. 105. 1884/85.

61. Ormerod, On hereditary locomotor ataxy. The brit. med, Journ. 1885. p. 435. Cit. Mendel's Centralblatt 1885. p. 382.

62. Botkin, Ein Fall von Friedreich'scher Krankheit. Medicinskoje Obosrenie 1885. No. 1. p. 32.

63. Seguin, Clinical Report of two sets Friedreichs Hereditary ataxy. Med. Record. New-York. 1885. XXVII. p. 645. Cit. Centralblatt für klin. Med. 1886. S. 39.

64. Palma, Malattia del Friedreich. Relazione sulla oura delle acque del Gurgitella praticata in Napoli. 1885.

65. Erlicki und Rybalkin, Wjestnik Psychiatrii. 1885. No. 1. Cit. Mendel's Centralblatt 1885 . S. 375 .

66. Fazio, Un altero caso della cosidetta atassia ereditaria o tabe del Friedreich. Rivista Clinica e terapeutica 1885. p. 73.

67. Krause, Zur pathologisohen Anatomie der Tabes dorsalis. Neurolog. Centralblatt 1885 . S. 69.

68. Charcot, L'ataxie héréditaire. Gazette des hôpitaux. 1888. - Baillarger, Annales médico-psychologiques 1885. p. 284.

69. Longuet, La maladie de Friedreich. Baillarger, Annales médicopsychologiques 1885 ..p. 302 .

70. Vizioli, La Malattia di Friedreicb. Naples Giorn. di Neuropatologia. 1885. Cit. Mendel's Contralbl. 1886. p. 111.

71. Remak, Drei Fälle von Tabes im Kindesalter. Berliner klin. Wochen schr. 1885 . No. 7. S. 105.

72. Morton Prince, Boston med. and surg. journ. CXIII. p. 371. Cit. Mendel's Centralbl. 1885. S. 537.

73. Kaymond, Tabes dorsalis et Tabes spasmodique. Dict. Encyclop. des sciences méd. Paris 1885.

74. E. Smith, Hered. or degenerative Ataxia. Boston med. and surg. journ. 1885. 15. Oct. Cit. Mendel's Centralbl. 1886. S. 536.

75. Ormerod, An account of two families screral membres of with ataxia. Med. chir. Trans. Vol. 68. 1885. p. 147. Cit. Mendel's Centralbl. 1885. S. 382.

76. Wharton Sinkler, Two cases of Friedreich's disease. Med. news. 1885. Juli 4. Cit. Mendel's Centralbl. 1886. S. 167. Centralbl. f. klin. Med. 1886. S. 40.

77. Mc Alister, Brit. med. Journal 1886. January 2. p. 19.

78. Charcot, Ueber bereditäre Ataxie. Neue Vorlesungen über die Krankheiten des Nerrensystems. Deutsche Ausgabe von Freud. 1886. S. 336 .

79. Judson S. Bury, A contribution to the symptomatology of Friedreich's disease. Brain. Vol, IV. 1886. p. 145. 
80. Erlicki und Rybalkin, Zur Frage über die combinirten Systemerkrankungen des Rückenmarkes. Dieses Archiv XVII. 1886. S. 692.

81. Schuster, Diagnostik der Rückenmarkskrankheiten. Berlin 1886. p. 97.

82. Seeligmüller, Krankheiten des Rückenmarks und Gehirns. 1886. S. 194.

83. Vierordt, Beitrag zur Kenntniss der Ataxie. Berliner klin. Wochenschrift 1866. S. 333 .

84. Lissauer, Beitrag zum Faserverlauf im Hinterhorn etc. Dieses Archiv Bd. XVII. 1886. S. 377. Neurol. Centralbl. 1885. No. 11.

85. Blocq, Un cas de maladie do Friedreich. Arch. de Neurol. 1887. p. 217. Cit. Mendel's Centrblatt 1887, S. 395. Centralbl. für klin. Med. 1888 . No. 8.

86. Rütimeyer, Ueber hereditäre Ataxie. Virchow's Archiv Bd. 110. S. 215. Cit. Mendel's Centralbl. 1888. No. 1. S. 25.

87. Mendel, Zur Lebre von der Ataxie. Berliner klin. Wochenschr. 1887. S. 771. Cit. Mendel's Centralbl. 1888. S. 26.

88. Descroizilles, Un eas d'ataxie héréditaire. Progr. méd. 1886. No.28. Cit. Centralbl. f. klin. Med. 1887. S. 199. Mendel's Centralbl. 1887. S. 394. Medicinische Bibliographie 1886. S. 308.

89. Ferrier, Case of Friedreich's disease. The brit. med. Journal 1887. June 4. p. 1214. Cit. Mendel's Centralbl. 1887. S. 396.

90. Stintzing, Ueber heriditäre Ataxio. Münchener med. Wochenschrift 1887. No. 21. Cit. Mendel's Centralbl. 1887. S. 396.

91. Freyer, Zur Tabes im jugendlichen Alter. Berliner klin. Wochensehr. 1887. S. 91.

92. Charcot, La maladie de Friedreich. Leçon de Charcot, recueillie par P. et H. Berbez. Progr. méd. 1887. No. 23. Cit. Mendel's Centralbl. 1887. S. 395. Gazette des hôpitaux. April 26. 1887.

93. Oppenheim und Siemerling, Beitr. zur Patohlogie der Tabes dorsalis etc. Dieses Archiv XVIII. 1887.

94. Gowers, Diseases of Spinal Cord. p. 349.

95. Dana, Progressive spastic ataxia etc. New-York med. Record. July 2. 1887.

96. Bristowe, System of Medicine.

97. Goodhard, Diseases of children.

98. Mendel, Zur Lehre von der Ataxie: Acute Bulbärparalyse - Friedreich'sche Tabes. Berliner klin. Woohenschrift 1887. No. 41. Cit. Mondel's Centralbl. 1888. S. 26.

99. Borgherini, Caso speciale di affezione combinata dei cordoni posteriori e laterali del midollo spinale. Rivista sperim. di freniatria. 1887. XIII. p. 137. Cit. Mendel's Centralbl. 1888. p. 300.

100. Ormerod, Some further observations on Friedreich's disease. Brain 1880. January. Cit. Mendel's Centralbl. 1888. S. 302.

101. Adamkiewioz, Ueber combinirte Degenerationen des Rückenmarks. 
VII. Congress für innere Medicin. Wiesbaden 9. bis 14. April 1.888; ref. Mondel's Centralbl. 1888. S. 272.

\section{Literatur über Kleinhirnatrophie.}

102. Weber, Verhandl. der Kaiserl. Leopold-Carol.-Academie. Bd. VI. I. Bonn 1828.

103. Combetti, Journal de physiol. par Magendie. XI. 1831. - Rerue médicale 1831. T. II. p. 57. Cit. b. Longet: Anatomio und Physiologio des Nervensystems; übers. $r$. Heine.

104. Turner, De l'atrophie unilatérale da cervelet etc. Thèse de Paris 1856. Baillarger: Annales médico-physiol. 1856. p. 453.

105. Fiedler (und Bergmann), Fall von Verkümmerung des Cerebellams. Zeitschr. f. rationelle Medic. von Henle und Pfeufer. Bd. XI. Heft 3. S. 250.1861 .

106. Duguet, Bulletin de la soc. anat. 1862. Janvier, Mai.

107. Crupeilhier, Anat. pathol. Livre XV. pl. V. Cit. Longet, Anat. et phys. etc. T. I. p. 764 .

108. Duguet et Vulpian, Deux cas d'atrophie du cervelet. Gaz. hebdom. 1862. No. 46.

109. Lallement, Bulletin de la soc. anat. 1862. Schmidt's Jahrbücher 1863. VIII.

110. Moreau de Tours, Denx cas de sclérose du cervelet. Gaz. des hôp. 1863. I.

111. Meynert, med. Jahrbücher 1864. IV. Heft. S. 108.

112. Clapton, Transactions of the pathol. society of London. $187 \mathrm{l}$.

113. Obersteiner, Eine partielle Kleinhirnatrophio etc. Allg. Zeitschr. f. Psych. u. gerichtl. Medicin. Bd. 27. 1871. S. 74.

114. Pierret, Note sur un cas d'atrophie eto. Arch. de physiol. norm. et pathol. 18.71/72. T. IV. p. 765. Cit. Baillarger, Annales médicopsychol. 1875. p. 474.

115. Otto, Dieses Archiv Bd. IV. und VI. 1873 und 1875.

116. Lubimoff, Virchow's Archir Bd. 57, 1873.

117. Andral, Clinique méd. III ód. V. Bd.

118. Verdelli, Rivista clinica. 1874. Maggio.

119. Fischer, Dieses Arohiv Bd. V. 1874.

120. Huppert, Hochgradige Kleinheit des Cerebellums. Dieses Arebiv Bd. 1876 . S. 98 .

121. Hitzig, Ziemssen's Handbuch XI. 1. 1878.

122. Nothnagel, Topische Diagnostik der Gehirnkrankheiten. Berlin 1879.

123. Seppilli, Fall von Atrophie des Kleinhirns. Riv. sperim. di freniatr. V. Bd. 4. Heft. 1879. Cit. Centralblatt für Nervenheilkunde. 1880. p. 180 . 
124. Fraser, Glasgow med. Journal 1880. 1. Heft.

125. Claus, Zur Casuistik der Erkrankungen des Centralnerronsysterns Dieses Archiv Bd. XII. S. 669. 1881. Cit. Mendel's Centralblatt' 1882. S. 327.

126. Bouillard, Nouvelles recherches etc. France médicale. 1882. Baillarger, Annales méd.-psychol. 1882.

127. Kirchoff, Ueber Atrophie and Sklerose des Kleinhirns. Dieses Archir Bd. XII. 1881.

128. Hitzig, Achte Wanderversammlung der südwestdeutschen Nourologen und Irrenärzte. Mendel's Centralbl. 1883. S. 292.

129. Borell, Achte Waderversammlung der südwestdeutschen Neprologen und Irrenärzte. Mendel's Centralbl. 1883. S. 292.

130. Herbert C. Mayor, Atrophie and Sclerosis of the cerebellum. Journal of mental soience 1883. Jan.

131. Sommer, Zur Casuistik der Kleinhirnsklerose. Dieses Archip Bd. XV. 1884.

132. Ingels, Arrêt de développement du cervelet chez un aliéné. Bulletin de la Soc. do méd. ment. de Belgique. 1884. No. 35. Cit. Mendel's Centralbl. 1885. S. 272.

133. Ferrier, The functions of the brain. II. ed. London 1886. Cap. VI. Cit. Mendel's Centralbl. 1887. S. 185.

134. Schultze, Ueber einen Fall ron Kleinhirnsohwund mit Degenerationen in verlängerten Marke and Rückenmarke. Virchow's Archiq Bd, 108. 1887.

135. Becker, Fall von hochgradiger Zerstörung des Kleinhirnwurmes nebst casuistischen Beiträgen zur Lehre von der sogenannten cerebellaren Ataxie. Virchow's Arohiv Bd. 114. 1888. S. 173.

136. Martinotti e Mercandino, Richerche sulle alterazioni del midollo spinale concomitanti le lesioni cerebellari. Il Morgagni. 1888. XXX. Heft 1. Cit. Mendel's Centralbl. 1888. S. 383.

137. Marchi, Sulle degenerazioni consecutive alla estirpazione totale $\theta \mathrm{n}$ ziale del cerveletio. Ripista sperim. di fren. 1888 . XIII. p. ${ }^{A}$.

\section{Erkärung der Abbildungen. ( $T$}

Fig. I. Schnitt in der Höhe des Austritter 
190 Dr. med. P. Menzel, Beitrag zur Kenntniss der hereditären Ataxie etc, wurzel.

Fig. II. Schnitt in der Höhe des Austrittes der sensiblen Trigeminus-

V. s. Sensible Trigeminuswurzel.

Brcj. Bindearm.

Py. Pyramiden.

f. Die Pyramiden nmgebende Fasern.

cb. Cerebellum.

V. IV. Vierter Ventrikel.

Fig. III. Transversalschnitt durch das Kleinhirn.

am. Amygdala.

c. d. Corpus dentatum.

x. wohlerhaltene Partie zwischen

1. l. p. lob. lunat. post. und

l. s. p. lob. super. post. s. semilunar.

1. o. lob. cuneiformis. 